\title{
What is the best strategy program to acquire video-assisted thoracoscopic surgery lobectomy technique?
}

\author{
Duilio Divisi, Gino Zaccagna, Roberto Crisci \\ Thoracic Surgery Unit, University of L’Aquila, “G. Mazzini” Hospital, Teramo, Italy \\ Correspondence to: Duilio Divisi, MD, PhD. Piazza Italia n.1, 64100 Teramo, Italy. Email: duilio.divisi@aslteramo.it. \\ Provenance: This is an invited article commissioned by the Section Editor Laura Chiara Guglielmetti (Cantonal Hospital Winterthur, Kantonsspital \\ Winterthur, Winterthur, Switzerland). \\ Response to: Dhanasopon AP, Boffa DJ. Video-assisted thoracoscopic surgery lobectomy: transitions in practice. J Thorac Dis 2018;10:S3834-6. \\ Konge L, Petersen RH, Ringsted C. Developing competency in video-assisted thoracic surgery (VATS) lobectomy. J Thorac Dis 2018;10:S2025-8.
}

Submitted Sep 27, 2018. Accepted for publication Nov 02, 2018.

doi: $10.21037 /$ jtd.2018.11.41

View this article at: http://dx.doi.org/10.21037/jtd.2018.11.41

\section{Introduction}

I would like to thank Konge et al. (1) for the interest and comment about my article. I take this opportunity to voice our point of view on the need of the specific videoassisted thoracoscopic surgery (VATS) lobectomies training program, whose methods focused on the different opinions of surgeons. While in the past VATS approach represented only the smallest part of total lobectomies, today this is around $90 \%$ of total interventions in high-volume centers. The new generations of surgeons are faced with the necessity to quickly acquire the mastery of this technique, with the best planning which still arouses debate and controversy among experienced colleagues.

\section{Comment}

Of course, it is commonly accepted that 50 procedures carried out in total autonomy is the minimum threshold to consider as having acquired the technical skills (2-4). On the contrary, the type of path to be followed in order to learn the technique in the best way is still controversial. Over the years, many virtual models have been developed for simulation in thoracic surgery that allow less experienced surgeons to practice and more experienced surgeons to evaluate the acquisition of skills. Useful tools with an important role in the training of beginners are simulators, virtual reality and cadaver labs $(5,6)$. Probably, simulators are the most realistic way to learn VATS lobectomies as demonstrated by Jensen et al. (7) which have put in place an extremely realistic simulation model that has been tested and evaluated by both novice surgeons and experienced open surgeons. Certainly, the simulator is a very useful training tool for young surgeons; nevertheless, presents limits in the training of senior surgeons. In fact, as the simulator is not yet equipped with software able to develop complex metrics that can be useful in the advanced training phase, the two categories of surgeons obtain overlapping results. An experienced surgeon will supposedly have a more highly developed technique compared to the less experienced novice. In practice, however, we have found equal difficulty in the two groups in the steps of methodology perhaps attributable to two factors; (I) the younger group due to greater skill (acquired with the use of the play station), are likely to have more fluidity of movement with video surgery; (II) as the simulator is not programmed for advanced surgical intervention, it allows for a uniform level of learning rendering it difficult to verify the true capacity of the individual surgeon. A further limitation of the simulators is that they are often very expensive and therefore not within the reach of all the centers. The difficulty of reproducing the human model leads the surgeons in training to resort to cadaver labs. In this case, the correspondence to reality is extremely high from an anatomical and practical point of view. However, even this practice has two types of limitations: (I) one of a purely technical nature, since the lack of blood circulation does not allow to realistically reproduce the vessels dissection; (II) the other, not less important, is characterized 
by the lack of these laboratories on the training network. Probably, the most valid alternative is represented by the ex-vivo perfused models such as the heart-lung animal block that are extremely realistic as compared to the simulation on a cadaver allowing to cannulate the pulmonary artery and making the dissection of vessels more accurate. The possibility of being re-used several times and to have a very low cost as they come from animals already used for other purposes, are the undoubted advantages (8). Obviously, all the simulation models have big limits and cannot replace "live" experience. In fact, as underlined by Konge et al. (9) the limitations are related to the non-perfect correspondence to reality and the lack of important scientific evidence to support its real effectiveness. We believe that the aspects to be taken into greater consideration are both the acquisition of the technique obtained by a theoretical path followed by practice in the operating room with the support of a tutor or a senior surgeon and the young surgeon's ability to manage complications that need conversion in open thoracotomy. In our recent work (2) we analyzed data from 1679 patients enrolled in the Italian Registry of VATS lobectomies to evaluate the parameters focused on during the training period. Among these, the interesting data emerged from the analysis related to the conversion rate that, in agreement with Petersen et al. (10), decreased as experience increased although this was maintained around $7-8 \%$ even at the end of the learning program. The outcomes suggest that there are situations in which the complication can only be managed with open conversion. We think that the consolidated experience in open surgery represents a "milestone" of the training program of VATS lobectomies, even though surgeons with little experience in open thoracotomy can achieve overlapping outcomes in the VATS resections to more experienced colleagues. We are the opinion that a deontologically correct approach to the patient imposes the capacity and autonomy management of every situation, predictable or not, with all methods available; for which the minimally invasive procedure for major resections must be a "second step" in the learning path or, in high-volume centers, contemporarily with the acquisition of the open technique. Leaving the obsolete posterolateral thoracotomy, the "first step" of learning should start from the muscle sparing axillary mini-thoracotomy with a diameter between 7 and $10 \mathrm{~cm}$ and from VATS minor resections. This progressive training path is the only one that can guarantee the total autonomy of performance of young surgeons, associated with ensuring patient safety when handling complex cases and complications.

\section{Acknowledgements}

None.

\section{Footnote}

Conflicts of Interest: The authors have no conflicts of interest to declare.

\section{References}

1. Konge L, Petersen RH, Ringsted C. Developing competency in video-assisted thoracic surgery (VATS) lobectomy. J Thorac Dis 2018;10:S2025-8.

2. Divisi D, Bertolaccini L, Barone M, et al. National adoption of video-assisted thoracoscopic surgery (VATS) lobectomy: the Italian VATS register evaluation. J Thorac Dis 2018;10:330-8.

3. Zhao H, Bu L, Yang F, et al. Video-assisted thoracoscopic surgery lobectomy for lung cancer: the learning curve. World J Surg 2010;34:2368-72.

4. Ra YJ, Ahn HY, Kim MS. Learning Curve of a Young Surgeon's Video-assisted Thoracic Surgery Lobectomy during His First Year Experience in Newly Established Institution. Korean J Thorac Cardiovasc Surg 2012;45:166-70.

5. Jensen K, Bjerrum F, Hansen HJ, et al. Using virtual reality simulation to assess competence in video-assisted thoracoscopic surgery (VATS) lobectomy. Surg Endosc 2017;31:2520-8.

6. Divisi D, Barone M, Zaccagna G, et al. Video-assisted thoracoscopic surgery lobectomy learning curve: what program should be offered in a residency course? J Vis Surg 2017;3:143.

7. Jensen K, Bjerrum F, Hansen HJ, et al. A new possibility in thoracoscopic virtual reality simulation training: development and testing of a novel virtual reality simulator for video-assisted thoracoscopic surgery lobectomy. Interact Cardiovasc Thorac Surg 2015;21:420-6.

8. Avila R, Achurra P, Tejos R, et al. Uniportal video-assisted thoracic surgery lobectomy using a novel perfused ex vivo simulation model. J Vis Surg 2016;2:155.

9. Konge L, Petersen RH, Hansen HJ, et al. No extensive experience in open procedures is needed to learn lobectomy by video-assisted thoracic surgery. Interact 
Cardiovasc Thorac Surg 2012;15:961-5.

10. Petersen RH, Hansen HJ. Learning curve associated with VATS lobectomy. Ann Cardiothorac Surg 2012;1:47-50.

Cite this article as: Divisi D, Zaccagna G, Crisci R. What is the best strategy program to acquire video-assisted thoracoscopic surgery lobectomy technique? J Thorac Dis 2018;10(12):E822-E824. doi: 10.21037/jtd.2018.11.41 